\title{
Detection and genetic analysis of feline immunodeficiency virus (FIVple) in southern African lions (Panthera leo)
}

\author{
Hayley R. Adams ${ }^{1,3^{*}}$, Moritz van Vuuren ${ }^{2}$, Anna Mari Bosman ${ }^{2}$, \\ Stephen Kania ${ }^{1}$ \& Melissa A. Kennedy ${ }^{1}$ \\ ${ }^{1}$ Department of Comparative Medicine, College of Veterinary Medicine, University of Tennessee, \\ 2407 River Drive, Knoxville, Tennessee 37996, U.S.A. \\ ${ }^{2}$ Department of Veterinary Tropical Diseases, Faculty of Veterinary Science, University of Pretoria, \\ Private Bag X04, Onderstepoort 0110, South Africa \\ Received 10 August 2010. Accepted 7 April 2011
}

\begin{abstract}
Feline immunodeficiency virus is a retrovirus of domestic cats that causes immunosuppressive disease and lifelong infection. Lentivirus has also been detected in African lions (Panthera leo). The lentivirus infecting lions in southern Africa has never been isolated; thus, knowledge about its molecular characteristics in these populations is limited. Our investigation used whole blood samples collected opportunistically from free-ranging southern African lions in Kruger National Park, South Africa, and from Hlane Royal National Park in Swaziland to analyse the lentivirus. Whole blood samples from captive exotic felids from zoos in South Africa and the United States, and from domestic cats in South Africa were analysed for comparison. A nested polymerase chain reaction (PCR) assay was used to amplify a portion of the proviral DNA encoding the reverse transcriptase. The nucleotide sequence of all products was determined and examined. The PCR assay was successful in amplifying lion lentivirus, with 52 positive and 65 negative samples. Of the 34 sequences amplified from a variety of felids, six showed an average of $96 \%$ homology to domestic feline lentivirus, and 28 showed an average of $94 \%$ homology to lion lentivirus. In addition, domestic cat lentivirus nucleic acid was amplified from a captive tiger, demonstrating the possibility of cross-species transmission.
\end{abstract}

Key words: feline immunodeficiency virus, Kruger National Park, lentivirus, lion, Panthera leo, PCR, South Africa.

\section{INTRODUCTION}

The virus family Retroviridae, of which lentiviruses are members, have been known for many years to infect a variety of domestic animals, including domestic cats. Lentiviruses are RNA viruses with high rates of mutation due to the lack of a proofreading enzyme (Pedersen et al. 1987; Goff et al. 2001). This results in a high error rate by the viral polymerase during DNA synthesis and leads to the rapid evolution of genetic variants during replication in the host (Doolittle et al. 1989; Gojobori et al. 1990; Johnson et al. 1991; Salminen et al. 1993). The feline immunodeficiency virus (FIV) was first discovered in domestic cats (Felis catus) with a history of chronic recurrent and opportunistic infections that resembled the immunodeficiency syndrome of humans with human immunodeficiency virus (HIV) infection. Feline immunodeficiency virus of domestic cats (FIVfca) causes a

\footnotetext{
*To whom correspondence should be addressed.

significant lifelong infection. Lentiviral infections have also been detected in several nondomestic feline species, including in African lion (FIVple) populations in eastern and southern Africa (Panthera leo), but significant sequence divergence exists among the feline lentiviruses of different host species, with as much as 30\% diversity in the conserved regions of the polymerase gene (Pol), and even higher diversity in the regions encoding the envelope glycoprotein and core protein (Brown et al. 1994; Langley et al. 1994; Carpenter et al. 1998; Terwee et al. 2005; Troyer et al. 2005). Diversity is also pronounced among the feline lentiviruses infecting a single nondomestic host species, where as much as a $20 \%$ divergence may exist in the Pol region among different isolates (Brown et al. 1994; Carpenter et al. 1998; Terwee et al. 2005; Troyer et al. 2005). These findings are in contrast to FIVfca, where viral subtypes, or clades, differ in their nucleotide sequences by only $5-10 \%$ across the entire 


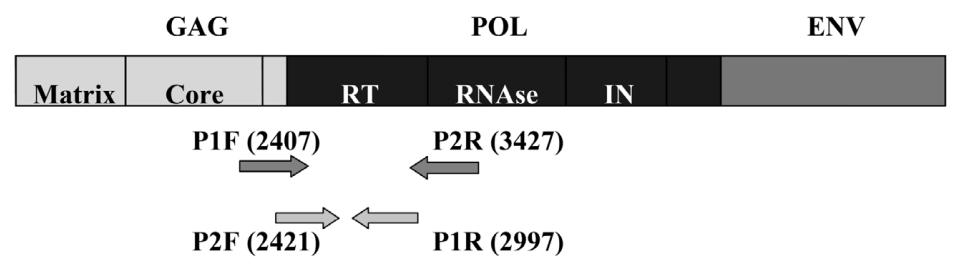

576 BP Product

Fig. 1. Schematic representation of the FIV genome, including the successful first and second round primers used and their nucleotide position within the RT-Pol region of the genome.

genome (Sodora et al. 1995; Carpenter et al. 1998).

Previous phylogenetic analyses of lion lentivirus Pol sequences in free-ranging lions revealed the presence of at least six clades (A-F) among lions in sub-Saharan Africa (O'Brien et al. 2006). While some of the subtypes have been found thus far in only one geographic region, such as subtypes $D$ in South Africa, E in Botswana, $C$ in Serengeti, and $F$ in Kenya, others have been sequenced from multiple geographic populations of free-ranging lions. For example, subtype A has been detected among the lions of RSA as well as among the lions of Botswana and Serengeti (O'Brien et al. 2006). All subtypes share a common monophyletic node, suggesting that FIVple infected a common ancestor early in the evolutionary history of the lion. Some viral subtypes then evolved in geographic isolation, while others found in multiple geographic locations may have evolved more recently as a result of lion translocations throughout Africa (O'Brien et al. 2006).

The lentivirus infecting lions in southern Africa has never been isolated, and few published studies exist on the epidemiology of the virus among free-ranging lion populations. As a result, little is known about the characteristics of the lentivirus in lions at the host and population level. The purpose of this study was to develop a polymerase chain reaction (PCR) assay for detection of FIVple from southern African lions, and to analyse the genetic relationship among different isolates from lions in the Kruger National Park (KNP), South Africa.

\section{METHODS}

\section{Sample collection and storage}

Seventy-three whole blood samples were available for evaluation from lions in KNP (KNP-Epi). The geographic locale within the park (north vs south) as well as the pride identity were used in the analysis. Also tested were whole blood samples from seven lions in The Lion Park, Johannesburg (LP), and whole blood from one lion in the St Louis Zoo (StL), United States. Finally, banked, frozen cell culture samples were tested and included 11 samples from KNP Mycobacterium tuberculosis-positive lions (KNP-Tb); eight samples from lions in the Hlane Game Reserve; samples from three lions in the Johannesburg Zoo (Jhb), samples from four tigers and three leopards in the National Zoological Gardens, Pretoria (Pta), and samples from seven domestic cats in Johannesburg (DC). Virus isolation was previously attempted by means of co-cultivation of Con A-stimulated peripheral blood mononuclear cells (PBMCs) from lentivirus seronegative and seropositive lions. Cell culture fluid containing the cell pellet was frozen pending further research. All whole blood and cell culture samples were stored at $-80^{\circ} \mathrm{C}$ until the time of processing and testing.

\section{DNA extraction and PCR}

DNA was extracted from whole blood and cell culture samples using the QIAGEN DNeasy Blood and Tissue Extraction kit (Invitrogen, Carlsbad, California) according to the manufacturer's directions. Nested conventional PCR was performed on the purified DNA, using a protocol developed by Troyer et al. for FIVple and optimized for use in testing southern African lions (Troyer et al., 2004; 2005). Primers were derived from GenBank published sequence information for FIVfca (M25381 and U11820), puma (Felis concolor) lentivirus (FIVpco) (U03982), and Pallas cat (Felis manul) lentivirus (FIVoma; U56928) by Troyer et al. (2004). The degenerate primers were developed to detect viral DNA from the RT-Pol (or reverse transcription region of $\mathrm{Pol}$ ) region of the lentivirus genome (Fig. 1), resulting in a nested 576 BP product (including nested primers). The $5^{\prime}$ and 3' outer oligonucleotide sequences were TGGCCWYTAWCWAATGAAAARATWGAAGC 
(referred to as P1F) and GTAATTTRTCTTCHG GNGTYTCAAATCCCC (referred to as P2R), respectively. The nested $5^{\prime}$ and $3^{\prime}$ oligonucleotides were TGAAAARATWGAAGCHTTAACAG AMATAG (referred to as P2F) and GTAATTTRT CTTCHGGNGTYTCAAATCCCC (referred to as P1R), respectively. All primers were synthesized by Sigma Genosys (Sigma, The Woodlands, Texas), and were reconstituted to a 20 pmol working solution.

The nested PCR was done using Platinum PCR SuperMix, containing $22 \mathrm{U} / \mathrm{ml}$ complexed recombinant Taq DNA polymerase with Platinum Taq Antibody, $22 \mathrm{mM}$ Tris- $\mathrm{HCl}$ at $\mathrm{pH} 8.4,55 \mathrm{mM}$ $\mathrm{KCl}, 1.65 \mathrm{mM} \mathrm{MgCl} 2,220 \mu \mathrm{M}$ concentrations of dGTP, dATP, dTTP, and dCTP (Invitrogen) with 20 pmol of each primer ( $F$ and $R$ ) and 5 I of DNA. The cycling conditions for the first round of PCR reaction consisted of a pre-denaturing step of three min at $94^{\circ} \mathrm{C}, 45$ cycles of $30 \mathrm{~s}$ at $94^{\circ} \mathrm{C}$ (denaturing), $30 \mathrm{~s}$ at $52^{\circ} \mathrm{C}$ (annealing), and $45 \mathrm{~s}$ at $72^{\circ} \mathrm{C}$ (extension), with a final extension for $10 \mathrm{~min}$ at $72^{\circ} \mathrm{C}$. Three microlitres of the resultant PCR product from round one were added to a nested PCR reaction mixture using $20 \mathrm{pmol}$ of the internal primers. The cycling conditions for the nested reactions were the same as for the first round PCR reaction. All reactions were carried out in an Eppendorf Mastercycler personal PCR thermocycler (Eppendorf, Westbury, New York). Products were analysed by agarose gel electrophoresis.

\section{PCR DNA purification and nucleotide sequencing}

DNA amplicons were extracted from agarose gel using the QIAGEN QIAquick gel extraction kit according to the manufacturer's instructions (Invitrogen) All positive PCR products were submitted for molecular sequencing at the University of Tennessee Molecular Biology Resource Facility DNA sequencing laboratory. The primers used for sequencing were P2F and P1R, at a five pmol solution.

Sequences were analysed using the National Center for Biotechnology Information Basic Local Alignment Search Tool (BLAST) software program and a search program for highly similar sequences (megablast). Phylogenetic analysis was performed on all sequences obtained using DNAstar Lasergene software.

\section{RESULTS}

The degenerate primers were developed to detect viral DNA from the RT-Pol (or reverse transcription region of Pol) region of the lentivirus genome, resulting in a nested $576 \mathrm{BP}$ product (including nested primers). Of the 117 samples tested with PCR, 52 were positive and 65 were negative for lion lentivirus. There were no positive samples from the KNP-Tb subset. Forty-four of 73 samples from KNP-Epi lions were positive and 29 were negative. Of the KNP-Epi samples, nine positives were from $23 \mathrm{KNP}$ North lions, and 35 positives were from $50 \mathrm{KNP}$ South lions. One Hlane lion was PCR positive out of the eight lions sampled. None of the lions tested from LP were positive. One of four tigers and all three leopards from the National Zoological Gardens in Pretoria tested positive, as well as the three lions from the Johannesburg zoo. Five of the seven domestic cat samples from Johannesburg tested positive by PCR. Finally, the St Louis lion tested positive for lion lentivirus by PCR. Figure 2 displays the positive bands illuminated after gel electrophoresis.

\section{Genetic and phylogenetic analysis}

Sequences were checked for homology using BLAST technology (see Appendix 1). All sequences amplified from the DC subset showed an average of $96 \%$ homology to FIVfca. All lion KNP sequences, as well as the St Louis lion, showed an average homology of $94 \%$ to FIVple. The viral sequence from a Pta tiger was $95 \%$ homologous to FIVfca, indicative of a cross-species infection. Sequences were placed in GenBank with accession numbers EF667006-EF667017, EF667019-EF667020, and EF667022-EF667041. A series of phylogenetic trees was constructed for the nucleotide sequences amplified from PCR (Figs 3 and 4). In general, clades were formed based on geographic location of north or south within the park, and were supported by high bootstrap values. There was some overlap between north and south clades, and overall, the KNP sequences shared an ancestral node supported by a high bootstrap value.

Figure 4 shows a phylogenetic tree of some KNP sequences randomly selected along with sequences from FIVple subtypes A-F, originally amplified from eastern African lions. Subtype A formed a clade with south KNP sequences; $D$ and $F$ formed a clade with a mix of south and north KNP sequences; subtypes $B$ and $C$ form a clade separate from all other sequences, showing a more distant phylogenetic relationship; and subtype E formed a clade with KNP north sequences. The domestic cat FIVfca sequences formed a clade that included the tiger FIVfca sequence, and 


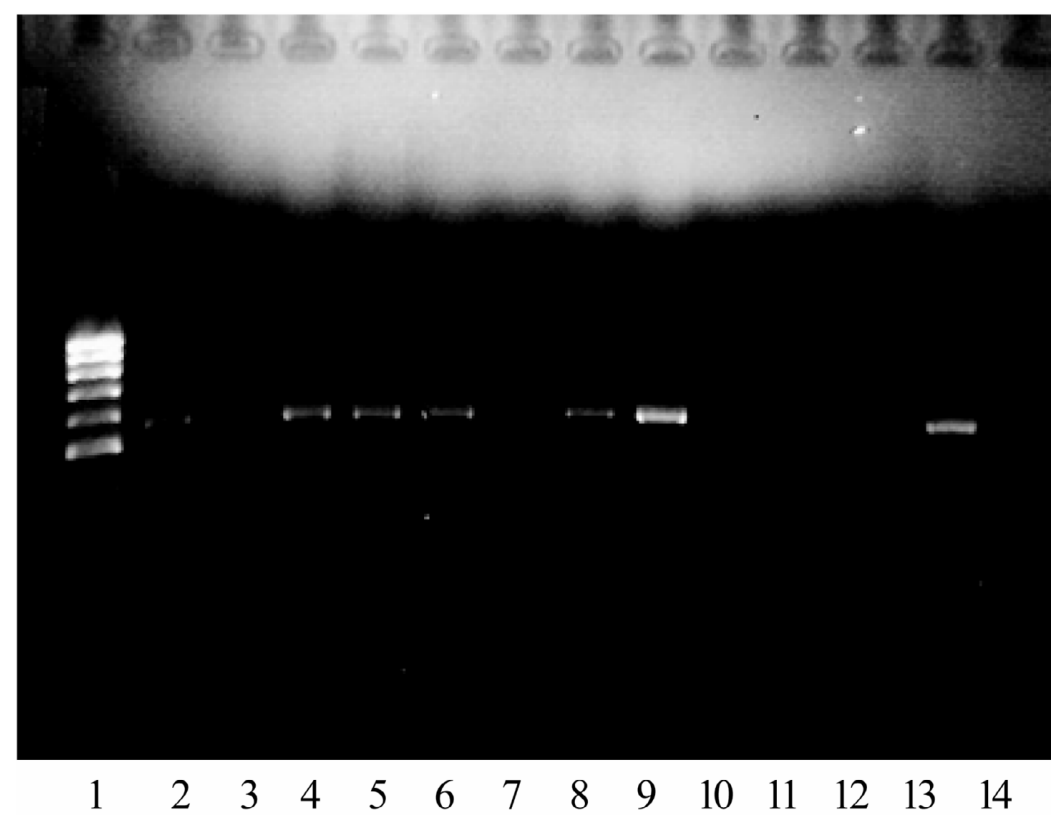

Fig. 2. 576 band size nested PCR products produced from P2F-P1R primers and visualized on a $1 \%$ agarose gel containing ethidium bromide. Band presence indicates a positive result. A $1 \mathrm{~Kb}$ ladder (lane 1) was used as a standard. Lanes $2-11$ are samples 1-10, respectively. Lane 12 was left blank; lanes 13 and 14 are positive and negative control samples, respectively.

the St Louis Zoo lion sequence formed a clade with KNP south isolates.

\section{DISCUSSION}

This investigation examined lentivirus infection in southern African felids and represents the first molecular epidemiological study of FIVple among the lions of the KNP, South Africa. The PCR assay used in this investigation utilized primers designed from published sequence information of the Pol-RT region of FIVfca, FIVpco, and FIVoma, and was successful in amplifying FIVple and FIVfca in the samples from southern African lions. The identity of the DNA products was confirmed by sequencing and comparison to other published FIV sequences. Previous studies have shown that lions in the Serengeti plains of eastern Africa have multiple subtypes circulating among their population, and some individual animals were infected with multiple subtypes (Troyer et al. 2004). The lions of KNP may also be infected with multiple subtypes of FIVple, some of which could not be detected with the PCR primers used in this study.

The feline lentiviruses have a high mutation rate and undergo continual evolution within a host and among a population in order to escape host immune defense mechanisms. This high mutation rate results in a variable population of virus subtypes that may in turn acquire new and distinct characteristics of virulence or pathogenicity. If lions are introduced to viral subtypes for which their immune systems have not co-adapted, an outbreak of disease is a potential outcome. In addition to lentiviral infections in lions, other infectious agents may play a role in shaping the overall immune competence of an individual, and within a population, which may in turn impact the response to infection with FIVple. Using PCR as an additional diagnostic test for FIV in lions will increase screening sensitivity and may help to identify which types and/or subtypes are circulating among a population of lions. In addition, it will be useful when translocations are necessary, in order to monitor for changes in the viral epidemiology of a region over time.

An overall pattern of phylogeography was established when looking at the FIVple sequences phylogenetically. Clades were formed in general based on geographic location in KNP (north vs south), with a common ancestral node, and were well-supported by a high bootstrap value. There was some mixing of sequences between the north and south, indicating the contiguous nature of the KNP ecosystem, allowing for the exchange of virus 


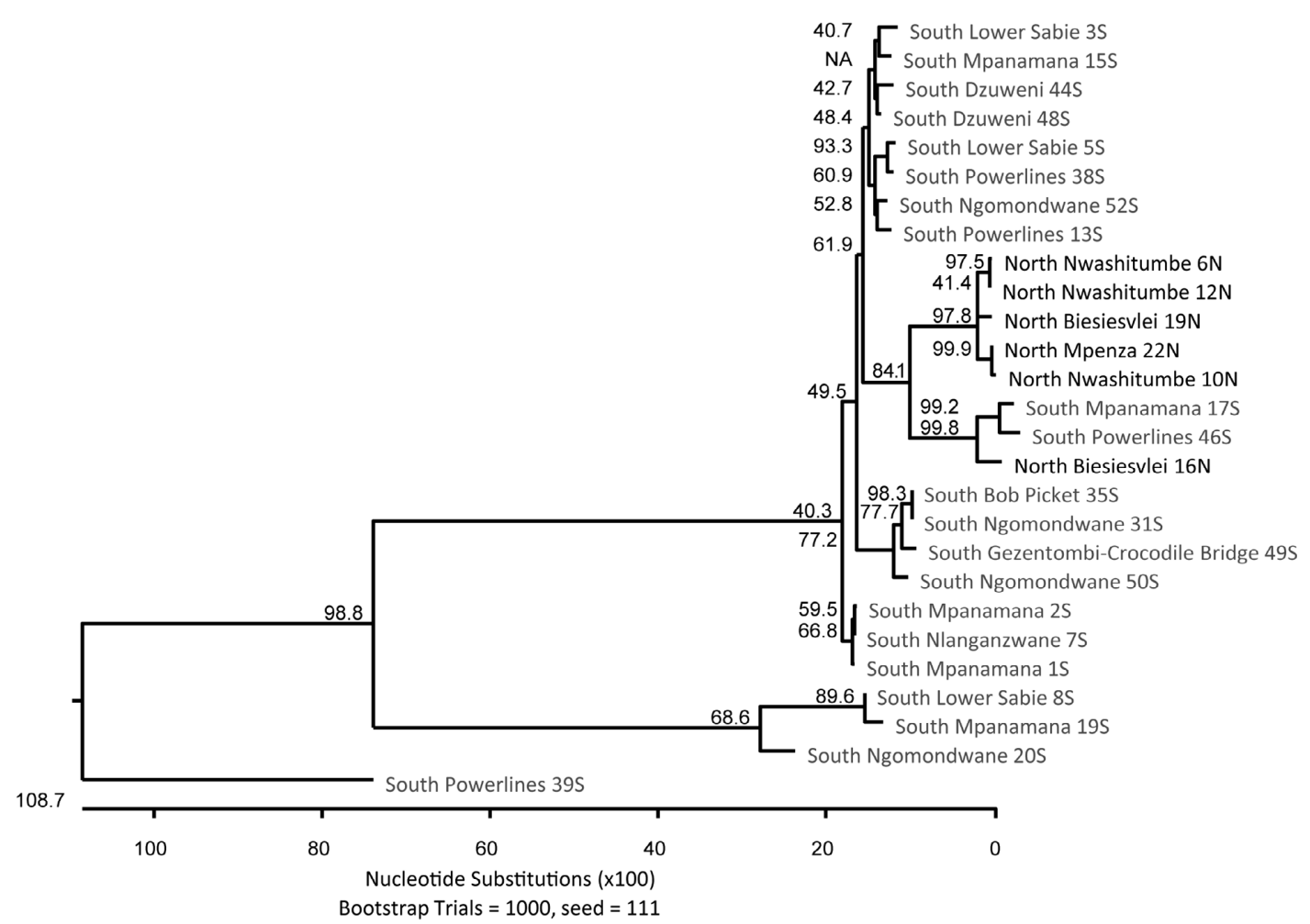

Fig. 3. RT-Pol phylogenetic tree of sequences from lions in the north and south sections of KNP, South Africa.

among the entirety of the KNP lion population. Although some sequence clustering existed by pride among clades, this was not a strict observance; some clades were composed of sequences from multiple prides of lions. This observance is plausible, given the fluid nature of lion social dynamics in KNP, where pride territories may overlap, and males roam freely from pride to pride.

Kruger National Park lion FIVple sequences were compared to those from previously characterized FIVple subtypes A-F. The isolates from lions in the north formed a clade with subtype $E$ (Botswana isolates), while the isolates from lions in the south formed a clade with subtype D (South African isolates). Subtype A (eastern and southern African isolates) formed a clade with a mix of both south and north KNP isolates. A clade composed of subtypes $B, C$, and $F$ (sequenced from lions in eastern Africa) was formed separate from all KNP isolates. It is possible that the subtypes shared among lions from more than one geographic locale, such as between eastern and southern Africa, either: 1) infected an ancestor of the modern African lion, and thus the subtypes share a deep ancestry as well; 2) infected African lions before more recent habitat fragmentation restricted lions to the geographic isolation seen today; 3) or infected lions in a new region as a result of translocation of infected lions, as part of repopulation efforts.

Several south KNP sequences formed a clade with a deep ancestral node separate from all other sequences, including all subtypes A-F. This may suggest the possibility of a subtype unique to the population of KNP lions, and more specifically to the lions of south KNP, that has not been previously identified. Alternately, it may indicate the presence of FIVple quasispecies in an individual lion host that differs substantially from other published amplified FIVple Pol sequences. It is interesting to note that the captive-born St Louis Zoo lion's FIVple sequence formed a clade with these sequences, which may indicate a genetic ancestry to the virus from lions of southern KNP. With these phylogenetic results, it is plausible that some or all of our seropositive/PCR-negative FIVple results may be attributed to a variety of subtypes and/or isolates present in KNP, not all of which were detected with the primers used in this study.

All domestic cat FIVfca sequences formed a 


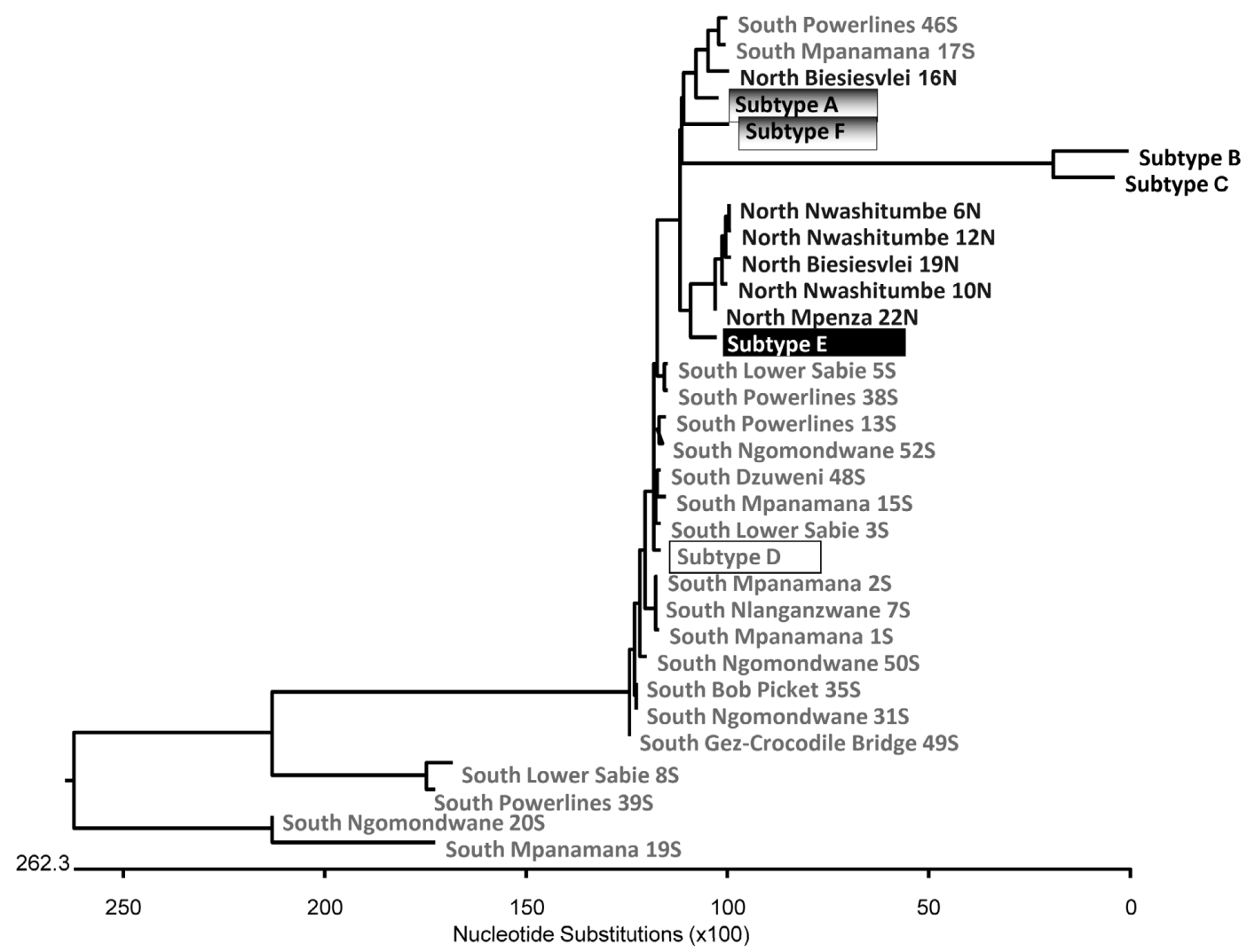

Fig. 4. RT-Pol phylogenetic tree of sequences from lions in KNP, South Africa. Reference RT-Pol NT sequences representing FIVple subtypes A-F, sequenced from eastern African lions, have been added for comparison. Eastern African subtypes: A (GenBank AY549255); B (GenBank AY552669); C (GenBank AY552683); D (GenBank AY878214); E (GenBank AY878235); and F (GenBank AY878220) sequences from Troyer (2004).

clade well supported by a high bootstrap value, along with a sequence amplified from a tiger at Pta, identified as an FIVfca isolate. This tiger's lentivirus infection is most likely due to a domestic cat transmission of FIVfca, such as a feral cat coming into direct contact with the captive felines of Pta.

We are only now beginning to understand the complex interplay that exists between the lion, its immune competence, and the co-evolution with FIVple that may or may not contribute to the manifestation of disease in some individuals. Unfortunately when dealing with free-ranging populations of wildlife, there is a limited opportunity to conduct long-term, exhaustive, invasive research into the effects of an infectious agent on a population; thus it is crucial to glean as much information as possible from the limited opportunities available for gathering data. Finally, lion conservation management would benefit from a more comprehensive knowledge of FIVple evolution, adaptation, and cross-species transmission for use when planning the development of a newly protected area, when planning strategies for the translocation of animals, as well as to plan for potential treatment and intervention strategies in the case of an outbreak of disease or other health crisis among a population.

The isolates sequenced in this investigation were only partial Pol nucleotide sequences and cannot be used to represent the viral evolutionary relationships as a whole. This can only be done once the entire FIVple genome has been sequenced for phylogenetic comparison. Other regions of the genome could also be sequenced in order to elucidate potential conserved areas ideal for a more universal PCR. The development of a realtime PCR assay would allow for the monitoring of viral subtype(s), viral load, and shedding patterns among individual free-ranging or captive FIVple positive lions, perhaps using non-invasive sampling techniques such as fecal samples. Much work 
remains in order to better characterize the lentiviruses of lions in general, and southern African lions in particular.

\section{ACKNOWLEDGEMENTS}

Funding was provided by the Center of Excellence in Livestock Diseases and Human Health and the College of Veterinary Medicine, University of Tennessee. Funding for sample collection and storage in South Africa was provided courtesy of the South Africa Veterinary Foundation. Samples from free-ranging lions were provided courtesy of the veterinary staff of South African National Parks - Kruger National Park Services. Samples from captive animals were provided courtesy of the National Zoological Gardens, Pretoria; the Johannesburg Zoo; The Lion Park, Johannesburg; and the St Louis Zoo, Missouri. Domestic cat samples were provided courtesy of Friends of the Cat, Johannesburg. We thank Kathy Thomas, Virology Laboratory, and Dianne Trent, Immunology Laboratory, of the Department of Comparative Medicine, The University of Tennessee; and Sandra Prinsloo, Serology Laboratory, Department of Veterinary Tropical Diseases, The University of Pretoria, for their laboratory assistance. Thanks are also due to Misty R. Bailey, Technical Communication Specialist, College of Veterinary Medicine, University of Tennessee, for assistance with manuscript editing, and to Anik Vasington, Communication Coordinator, College of Veterinary Medicine, University of Tennessee, for assistance with figure editing.

\section{REFERENCES}

BROWN, E., YUHKI, N., PACKER, C. \& O'BRIEN,S. 1994. A lion lentivirus related to feline immunodeficiency virus: epidemiologic and phylogenetic aspects. J. Virol. 68: 5953-5968.

CARPENTER, M., BROWN, E., MACDONALD, D. \& O'BRIEN, S. 1998. Phylogeographic patterns of feline immunodeficiency virus genetic diversity in the domestic cat. Virology 251: 234-243.

DOOLITTLE, R., FENG,D., JOHNSON, M. \& McCLURE,
M. 1989. Origins and evolutionary relationships of retroviruses. Quart. Rev. Biol. 64: 1-30.

GOFF, S. 2001. Intracellular trafficking of retroviral genomes during the early phase of infection: viral exploitation of cellular pathways. J. Gene Med. 3: 517-528.

GOJOBORI, T., MORIYAMA, E. \& INA, Y. 1990. Evolutionary origin of human and simian immunodeficiency viruses. P. Natl. Acad. Sci. USA 87: 4108-4111.

JOHNSON, P. \& HIRSH,V. 1991. Pathogenesis of AIDS: the non-human primate model. AIDS. 5 Suppl. 2: S43-48.

LANGLEY, R., HIRSCH, V., O'BRIEN, S., ADGERJOHNSON, D, GOEKEN, R. \& OLMSTED, R. 1994. Nucleotide sequence analysis of puma lentivirus (PLV-14): genomic organization and relationship to other lentiviruses. Virology 202: 853-864.

O'BRIEN, S.J., TROYER, J.L., ROELKE, M., MARKER, L. \& PECON-SLATTERY, J. 2006. Plagues and adaptation: lessons from the Felidae models for SARS and AIDS. Biol. Conserv. 131: 255-267.

PEDERSEN, N., HO, E., BROWN, M. \& YAMAMOTO, J. 1987. Isolation of a T-lymphotropic virus from domestic cats with an immunodeficiency-like syndrome. Science 235: 790-793.

SALMINEN, M., NYKANEN, A., BRUMMER-KORVENKONTIO, H., KANTANEN, M., LIITSOLA, K. \& LEINIKKI, P. 1993. Molecular epidemiology of HIV-1 based on phylogenetic analysis of in vivo gag p7/p9 direct sequences. Virology 195: 185-194.

SODORA, D., COURCELLE, J. \& BROJATSCH, J. 1995. Analysis of a feline immunodeficiency virus provirus reveals patterns of gene sequence conservation distinct from human immunodeficiency virus type 1 . AIDS Res. Hum. Retrov. 11: 531-533.

TERWEE, J., YACTOR, J., SONDGEROTH, K. \& VANDEWOUDE, S. 2005. Puma lentivirus is controlled in domestic cats after mucosal exposure in the absence of conventional indicators of immunity. J. Virol. 79: 2797-2806.

TROYER, J., PECON-SLATTERY, J., ROELKE, M., BLACK, L., PACKER, C. \& O'BRIEN, S. 2004. Patterns of feline immunodeficiency virus multiple infection and genome divergence in a free-ranging population of African lions. J. Virol. 78: 3777-3791.

TROYER, J., PECON-SLATTERY, J. \& ROELKE, M. 2005. Seroprevalence and genomic divergence of circulating strains of feline immunodeficiency virus among Felidae and Hyaenidae species. J. Virol. 79: 8282-8294. 
Appendix 1. BLAST results for all nucleotide sequences. Sequence homology and GenBank accession IDs for closest nucleotide sequence also provided.

\begin{tabular}{|c|c|c|c|}
\hline Sample ID & $\begin{array}{l}\text { Virus identity } \\
\text { (BLAST) }\end{array}$ & $\begin{array}{c}\text { Sequence homology } \\
(\%)\end{array}$ & $\begin{array}{l}\text { GenBank accession no. for closest } \\
\text { NT sequence homology }\end{array}$ \\
\hline DC Joburg 1D & FIVfca Australia & 96 & L16942 \\
\hline DC Joburg 3D & FIVfca Switzerland & 95 & X57002 \\
\hline DC Joburg 4D & FIVfca San Diego & 96 & M36968 \\
\hline DC Joburg 5D & FIVfca Australia & 96 & L16942 \\
\hline DC Joburg 6D & FIVfca Australia & 96 & L16942 \\
\hline KNP-N Bv 16 & FIVple & 93 & AY878216 \\
\hline KNP-N Bv 19 & FIVple & 91 & AY878208 \\
\hline KNP-N Mp 22 & FIVple & 89 & AY878208 \\
\hline KNP-N Nw 6 & FIVple & 90 & AY878235 \\
\hline KNP-N Nw 10 & FIVple & 90 & AY878208 \\
\hline KNP-N Nw 12 & FIVple & 90 & AY878235 \\
\hline KNP-S BP 35 & FIVple & 95 & AY878224 \\
\hline KNP-S Dz 44 & FIVple & 91 & AY878214 \\
\hline KNP-S Dz 48 & FIVple & 96 & AY878214 \\
\hline KNP-S Gez 49 & FIVple & 94 & AY878224 \\
\hline KNP-S LS 3 & FIVple & 96 & AY878214 \\
\hline KNP-S LS 5 & FIVple & 96 & AY878214 \\
\hline KNP-S LS 8 & FIVple & 92 & AY878214 \\
\hline KNP-S Mp1 & FIVple & 98 & AY878224 \\
\hline KNP-S Mp2 & FIVple & 98 & AY878224 \\
\hline KNP-S Mp 15 & FIVple & 96 & AY878214 \\
\hline KNP-S Mp 17 & FIVple & 96 & AY878216 \\
\hline KNP-S Mp 19 & FIVple & 95 & AY878214 \\
\hline KNP-S Ng 20 & FIVple & 86 & AY878224 \\
\hline KNP-S Ng 31 & FIVple & 95 & AY878224 \\
\hline KNP-S Ng 50 & FIVple & 95 & AY878224 \\
\hline KNP-S Ng 52 & FIVple & 96 & AY878214 \\
\hline KNP-S Nz 7 & FIVple & 99 & AY878224 \\
\hline KNP-S PL 13 & FIVple & 95 & AY878214 \\
\hline KNP-S PL38 & FIVple & 95 & AY878214 \\
\hline KNP-S PL 39 & FIVple & 96 & AY878224 \\
\hline KNP-S PL 46 & FIVple & 92 & AY878216 \\
\hline St Louis Zoo 53 & FIVple & 92 & AY549255 \\
\hline Pta Tiger 13 & FIVfca-US & 95 & M25381 \\
\hline
\end{tabular}

